\title{
Scanning Photoelectron Microscopy: a Powerful Technique for Probing Micro and Nano-Structures*
}

\author{
Majid Kazemian Abyaneh, ${ }^{\dagger}$ Luca Gregoratti, Matteo Amati, Matteo Dalmiglio, and Maya Kiskinova \\ Sincrotrone Trieste S.C.p.A., in Area Science Park, \\ SS-14, Km 163.5, 34149 Bassoviza, Trieste, Italy
}

(Received 12 November 2010; Accepted 25 February 2011; Published 9 April 2011)

\begin{abstract}
Scanning photoelectron microscopy (SPEM) is a powerful technique to image and probe micro and nanostructures. Recent achievements on imaging the sub-micro objects will be present in this report. For the first time, SPEM is used to explore and probe the chemical state and chemical composition of individual ZnO nanostructures. The capability of SPEM on imaging an individual MWCNT is shown which is down to $50 \mathrm{~nm}$ in diameter. The MWCNTs, partially covered with a metal, successfully are fabricated and presence of a sharp interface between CNT and metal is confirmed by SPEM. The morphology, composition and oxidation/reduction of isolated supported PtRh micro- and nano- particles produced by pulsed-laser deposition (PLD) have been investigated by SPEM. [DOI: 10.1380/ejssnt.2011.158]
\end{abstract}

Keywords: Scanning photoelectron microscopy (SPEM); Multi-wall carbon nanotube; XPS; Spectromicroscopy

\section{INTRODUCTION}

Nanoscience and nanotechnology have attracted tremendous interest during the last few decades and the advancement of nanomaterials science is quite rapid. It is mostly owing to the various microscope facilities which capable scientist to see and probe the nanostructures.

The imaging and analytical capabilities, combined with the latest developments of sample stages in these machines, have allowed in situ exploration of the mechanical, compositional, electrical, and other properties of the nanostructures, as well as high-resolution imaging and analysis at elevated pressures and temperatures. Different electron microscopy techniques, like scanning electron microscopy (SEM) and transmission electron microscopy (TEM) provide very high spatial resolution, but their spectroscopic abilities, based on energy-filtered electron and photon emission, such as electron energy loss, auger electron and energy dispersive X-ray spectroscopy, are limited in energy resolution and/or surface sensitivity. Alternative spectromicroscopy methods with surface sensitivity are those using X-ray sources and detecting photoelectrons emitted from the top few layers of the specimen. Probing the structures at submicrometer length scales using these techniques requires the intense and tunable Xrays provided at the third generation synchrotron facilities. The spatial resolution is achieved using two different approaches, which classify the microscopies as scanning and full-field imaging $[1,2]$. In the scanning photoelectron microscopy (SPEM), the incident photon beam is demagnified to a submicrometer ( $c a$. 100-200 nm) spot onto the sample, which can be raster scanned with respect to the microprobe. In the X-ray photoelectron emission microscopies (XPEEM), a magnified image of the irradiated area is obtained by projection of the emitted electrons using appropriate electron tailoring optics. These synchrotron-based microscopes can be successfully com-

\footnotetext{
* This paper was presented at the 6th International Workshop on Nano-scale Spectroscopy and Nanotechnology (NSS-6), Kobe University Centennial Hall, Kobe, Japan, 25-29 October, 2010.

†Corresponding author: majid.kazemian@elettra.trieste.it
}

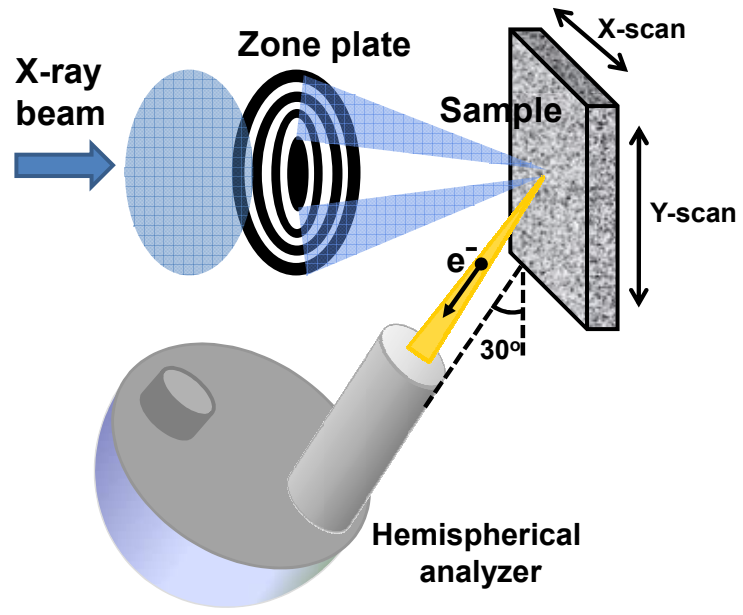

FIG. 1: Schematic diagram of the scanning photoelectron microscope (SPEM).

plemented with conventional surface science methods for structural analysis.

In this report, we present some recent experiments which carried out using SPEM. It provides new important information for various micro and nano-materials.

\section{EXPERIMENTAL AND CHARACTERIZATION TECHNIQUES}

SPEM experiments were performed at the ESCAmicroscopy beamline at the Elettra synchrotron radiation facility located in Trieste, Italy. Synchrotron source Xray beam is focused on the sample with a diameter of around $150 \mathrm{~nm}$ using Fresnel zone plate optics (Fig. 1). In SPEM the sample can be raster scanned with respect to the microprobe. Photoelectrones are collected with a SPECS-PHOIBOS 100 hemispherical analyzer and detected by a 48-chanel electron detector [3]. The position of the electron analyzer with respect to the sample surface is fixed at 30 degrees. This configuration strongly enhances the surface sensitivity of the instrument. The microscope operates in imaging and spectroscopic modes. 


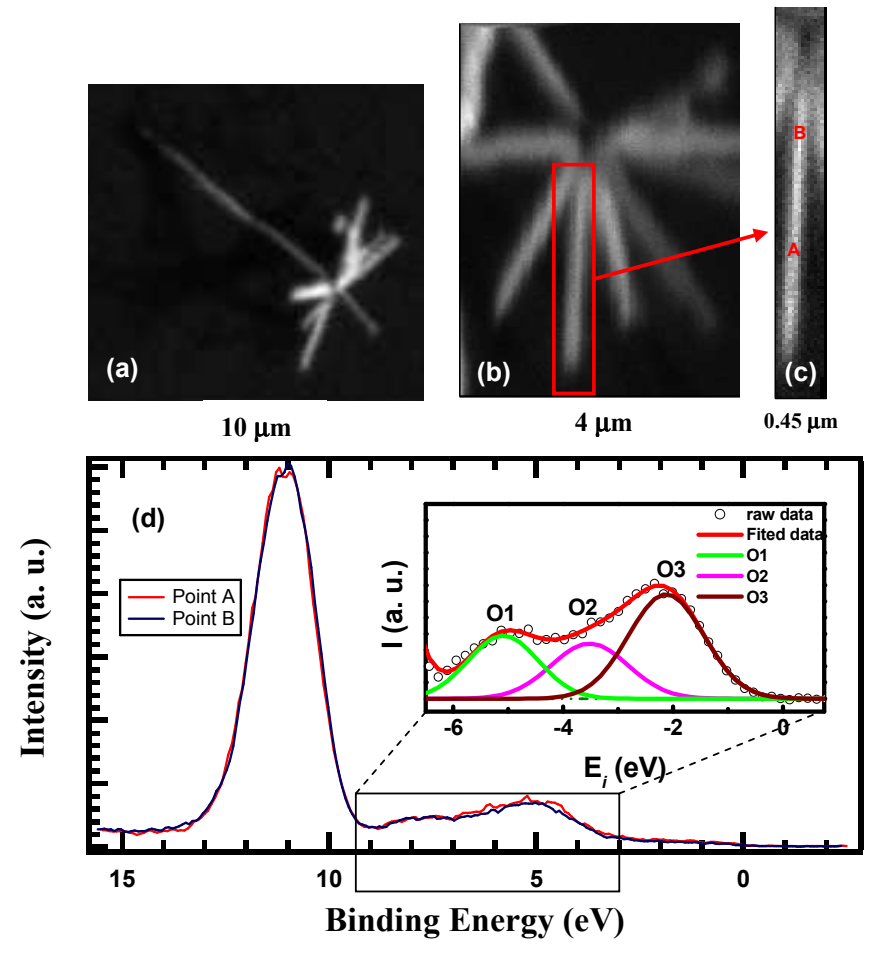

FIG. 2: (a) and (b) SPEM images of $\mathrm{ZnO}$ multipod structures, (c) SPEM image of the selected branch of $\mathrm{ZnO}$ structure and (d) XPS spectra ( $\mathrm{Zn} 3 d$ ) of $\mathrm{ZnO}$ branch corresponding to the assigned points on the branch. The inset shows the valence $\mathrm{O}$ $2 p$-bands in details assigned as $\mathrm{O} 1, \mathrm{O} 2$ and $\mathrm{O} 3$. The $E_{i}=0$ corresponds to the edge of the valence band.

Spatially resolved photoemission spectra of selected regions and chemical maps were acquired with $0.2 \mathrm{eV}$ energy resolution by using $650 \mathrm{eV}$ photon energy. SPEM can be operated in two modes: (i) imaging spectromicroscopy and (ii) microspot spectroscopy. The imaging mode maps the lateral distribution of elements by collecting photoelectrons with a selected kinetic energy window while scanning the specimen with respect to the microprobe. When the element under consideration is present in a single chemical state, the spatial variation in the contrast of the images reflects the variation of the photoelectron yield, which is a measure of the local concentration of the element. The microspot mode is identical to the conventional X-ray photoelectron spectroscopy (XPS), i.e. energy distribution curves are measured from the illuminated local micro-spot area.

\section{RESULTS AND DISCUSSIONS}

In this section, we would like to present some recent SPEM experiments which are performed on micro and nano structures. We will interpret the trends which occur to improve the spatial resolution of the SPEM to probe nanostructures in specific manner.

\section{A. Multi-pod $\mathrm{ZnO}$ structures}

The first example is to investigate the synthesized prickly and multi-pod shape $\mathrm{ZnO}$ nanostructures by SPEM. For the first time, scanning photoelectron microscopy (SPEM) is used to explore and probe at submicrometer length scale the chemical state and composition of the $\mathrm{ZnO}$ nanostructures. SPEM is a novel tool for characterization of the individual nano-objects. The nanostructures of $\mathrm{ZnO}$ have been obtained by the hydrothermal synthesis technique [4]. ZnO structures are analyzed using photoelectron spectromicroscopy which are shown in Fig. 2. The SPEM images of a multi-pod $\mathrm{ZnO}$ structure are shown in Figs. 2(a) and (b) which are taken by monitoring the $\mathrm{Zn} 3 d$ core level emissions and the contrast variations convey both morphological and compositional information. Specifically, the brighter and darker areas can result from: (i) topography-related enhancement, (ii) shadowing of the electron emission or (iii) regions exhibiting different chemical composition. The image of Fig. 2(c) shows a magnified image of a branch of $\mathrm{ZnO}$ structure. Using this image one can select different point on the structure to take the XPS spectrum. Figure $2(\mathrm{~d})$ exhibits the photoelectron spectrum of the $\mathrm{Zn}$ $3 d$ and valence $\mathrm{O} 2 p$-bands for points $\mathrm{A}$ and $\mathrm{B}$ on the $\mathrm{ZnO}$ structure shown in Fig. 2(c). The inset in Fig. 2(d) shows the valence $\mathrm{O} 2 p$-bands which are deconvoluted to three components. The valence band analysis revealed that these peaks are attributed to the bonding, nonbonding and antibonding of the zinc and oxygen orbitals. The O1, O2 and O3 peaks are three components of valence O $2 p$-bands where their relative intensities depend on the photon energy. It is believed that the O1 peak corresponds to the bonding $\mathrm{O} 2 p+\mathrm{Zn} 4 s+\mathrm{Zn} 4 p$-orbitals, the $\mathrm{O} 2$ peak attributed to the nonbonding "pure" O $2 p$-states and to the $\mathrm{O} 2 p$-states with small admixtures of the $\mathrm{Zn}$ $4 p$ - and $\mathrm{Zn} 3 d$-states. and the $\mathrm{O} 3$ peak is due to the antibonding $\mathrm{O} 2 p+\mathrm{Zn} 3 d$-orbitals [5]. These $\mathrm{ZnO}$ structures exhibit intense near band-edge emission. Photoluminescence analysis showed that the structures also give defectinduced blue emission along with green emission [4]. The photoluminescence (PL) spectrum of $\mathrm{ZnO}$ nanoarchitectures demonstrates that the photon excitation of the sample interestingly gives two blue-emission peaks at energies of $2.82,2.62 \mathrm{eV}$ and one broad green-band around $2.38 \mathrm{eV}$. It is believed that the blue-emission bands are attributed to the zinc vacancy and interstitial zinc and the greenemission peak is due to antisite oxygen. The luminescence property of these $\mathrm{ZnO}$ nanostructures preserved them potentially useful for a wide variety of applications in optical devices and optoelectronics. The novel morphology and compositional homogeneity of the synthesized $\mathrm{ZnO}$ nanoarchitectures make them a good candidate for use in various applications such as solar cells, superhydrophobic coatings, energy conversion/harvesting, chemical sensing, etc.

\section{B. Multi-wall CNTs}

The second example is about our recent achievements in imaging of multi-wall carbon nanotubes (MWCNTs) us- 
ing SPEM. The unique electronic, mechanical and transport properties of the CNTs due to the covalent $s p^{2}$ bonds and tubular structure with large length/diameter ratios introduce them as excellent candidates for many potential applications. CNTs have found great interests and applications in various devices like electronic devices, sensors, actuators, field emitters, energy storage media, etc. The high stability of the CNTs in an oxidative environment renders them not only excellent catalyst support materials but also high-performance catalysts for hydrocarbon oxidation [6]. Moreover, their adaptive functionalization by adsorption of foreign atoms have made them a key material in nanotechnology $[7,8]$.

We have already studied the oxidation of CNTs [9] and carried out many experiments to investigate the mass transport of metals deposited on the CNTs [10]. At the present, we are able to take SPEM image of CNTs down to $50 \mathrm{~nm}$ in diameter. The spatial resolution of SPEM in our beamline is high, but is limited down to $150 \mathrm{~nm}$ due to photon spot size. The goal was to image CNTs less than $150 \mathrm{~nm}$. To achieve this goal, we have used the low density aligned CNTs which allow us to detect the ejected photoelectrons from the CNTs even if the spot size is bigger than the NTs diameter. Figure 3(a) exhibits a typical SPEM image of aligned MWCNTs which have grown vertically on a Si substrate [11]. The image is taken for C $1 s$ core level, hence the CNTs appear in bright contrast and the vacuum background appears as dark contrast. In this image, the MWCNTs length is around $10 \mu \mathrm{m}$ and their tip diameter is around $70 \mathrm{~nm}$. Figure 3(b) shows the SPEM images of an individual MWCNT which is partially covered with gold at the middle. The two images were taken at $\mathrm{C} 1 s$ and $\mathrm{Au} 4 f$ core level energies. The XPS spectra which are taken inside and outside of the gold patch confirm the presence of the gold inside the patch. Gold is thermally evaporated on middle of an aligned multiwalled CNTs, $10 \mu \mathrm{m}$ long with an average external diameter of about $100 \mathrm{~nm}$ and grown on a Si substrate. We are able to deposit in-situ and partially, any metal on the CNTs. This technique facilitates to have sharp board between metal and CNT surface which can help to study properly the transport properties of CNTs. Figure 3(c) shows a C $1 s$ SPEM image that exhibits an individual MWCNT located on a $\mathrm{SiO}_{2}$ substrate and four gold electrodes which are deposited on the CNT using electron-beam lithography. This configuration provides a real electronic device that can be used for measurements in real working condition. These experiment are going on in our labs.

\section{Oxidation of PtRh nanoparticles with atomic oxygen}

Extensive studies of oxidation/reduction of $\mathrm{Pt}$ and $\mathrm{Rh}$ single crystals and vicinal surfaces have been performed following a bottom-up approach aimed to bridge the "material gap" between model catalysts and supported metal particles [12]. The observation of stoichiometric bulk oxide formation mediated via surface one or the morphological changes occurring on vicinal surfaces rich of undercoordinated atoms at the step edges demonstrate the complexity of the processes and the difficulty to extend the results to real systems where supported particles of

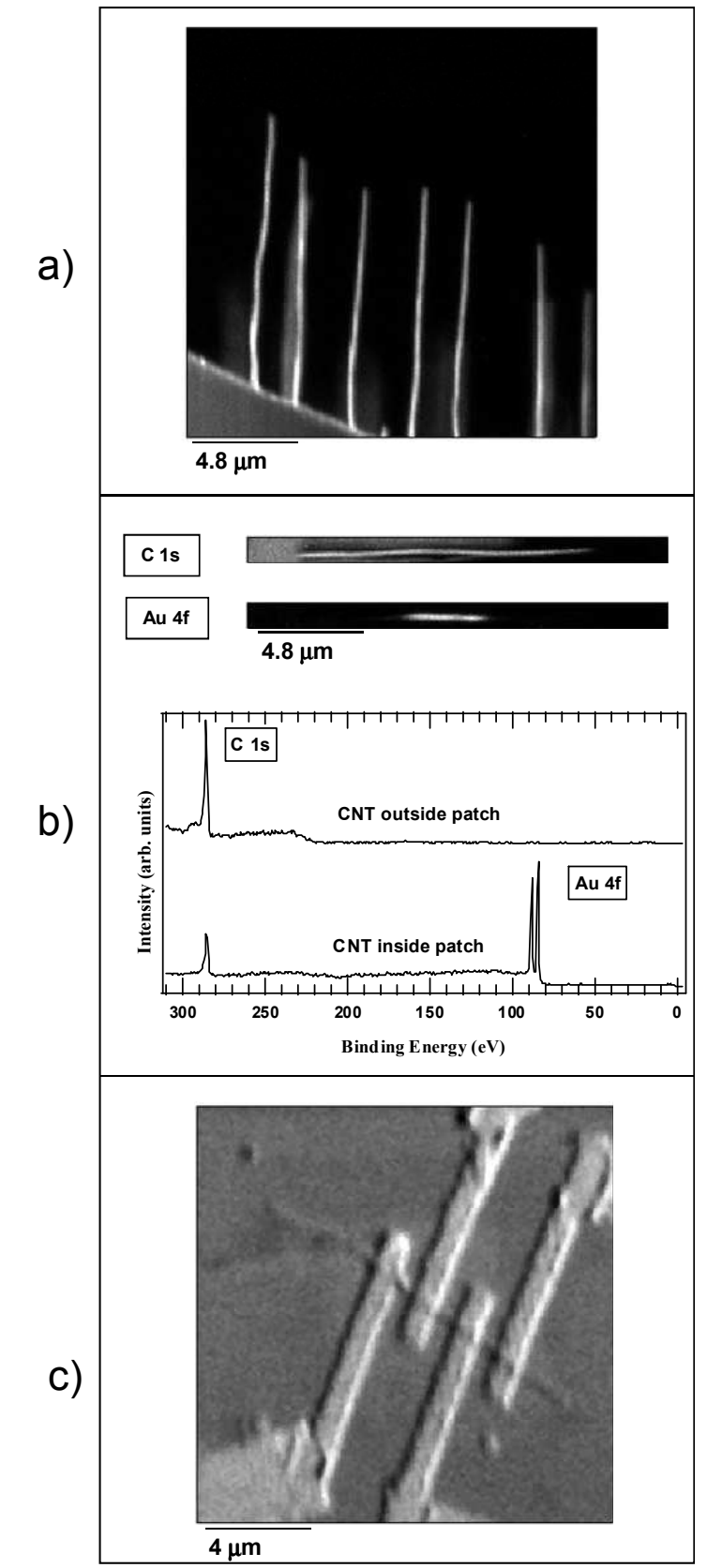

FIG. 3: (a) SPEM image of the vertically aligned multiwall CNTs. (b) SPEM images of partially gold metal coated MWCNT and the XPS spectra taken inside and outside of the Au patch. (c) SPEM image of an individual MWCNT with four-probe electrode installed on a chip for in-situ measurements.

different size and shape are used instead of extended ordered surfaces. The influence of the surface structure and atomic composition on the catalytic performance is less predictable when dimensionality is reduced as demonstrated by several studies $[13,14]$. Moreover the size of the particles in real catalytic converters can vary over orders of magnitude. The majority of the clusters being very small ones characterised by an appreciable number of coordinatively unsaturated atoms at the surface, promoting surface stresses and elevated atomic mobilities at the surface and larger particles where quantum size and 


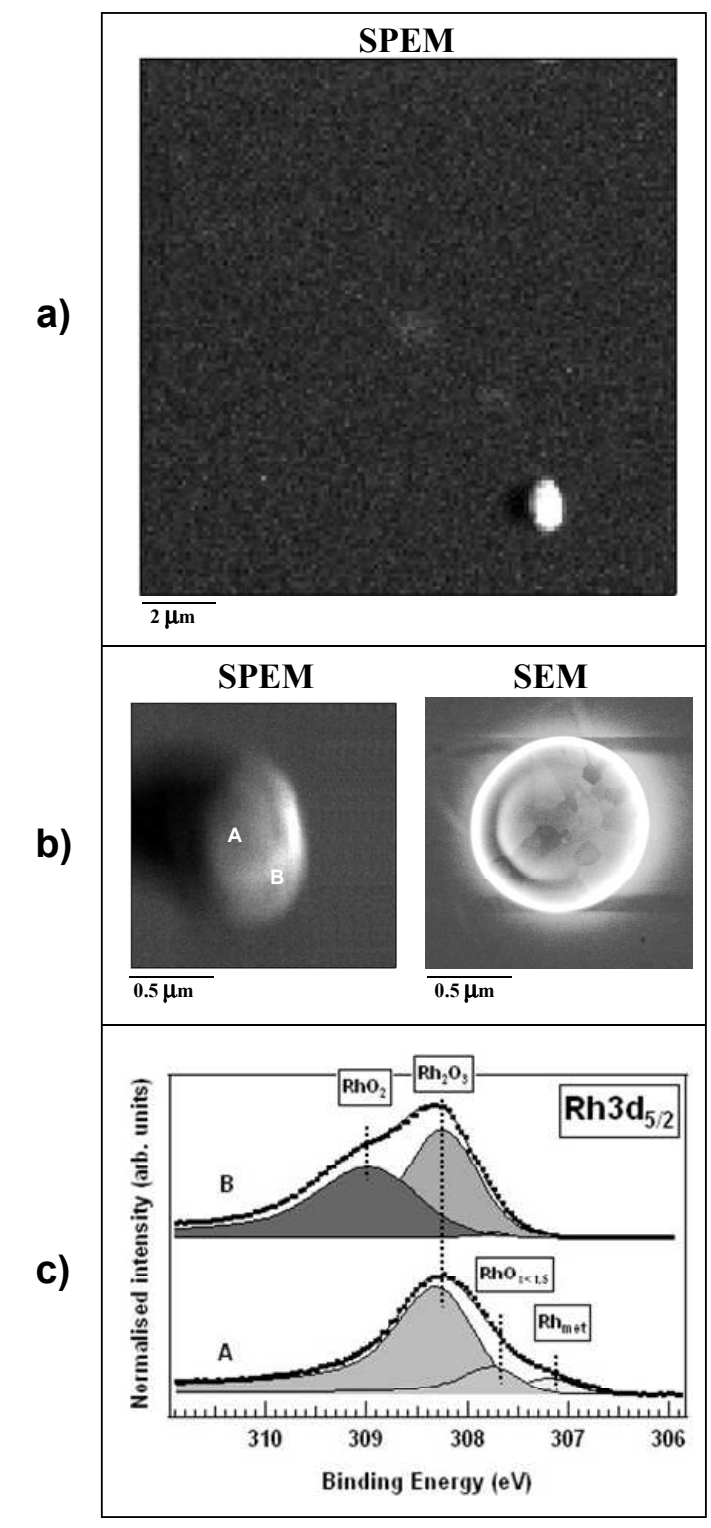

FIG. 4: a) SPEM Rh map showing a single isolated micronsized PtRh particle. The $10 \%$ of the surrounded area is covered by PtRh nanosized particles not visible with the lateral resolution achievable. b) SPEM and SEM images of typical PtRh particles produced with PLD. c) Rh $3 d_{5 / 2}$ spectra acquired in points $\mathrm{A}$ and $\mathrm{B}$ as indicated in b) after $715^{\prime}$ exposure of atomic oxygen (dose of $\sim 4 \times 10^{17}$ at. $\mathrm{cm}^{2}$ ). The energy positions of the main components considered in deconvolution of the spectra are indicated with dashed lines. For deconvolution of the Rh spectra the well-developed procedure with DoniachSunjic functions convoluted with Gaussians was used.

support interfacial effects are less important.

The model supported catalysts were prepared by using the Pulsed Laser Deposition (PLD) ablating a $\mathrm{Pt}_{50} \mathrm{Rh}_{50}$ target with a Nd:YAG laser operating at $532 \mathrm{~nm}$ wavelength with a fluence of approximately $10 \mathrm{~J} / \mathrm{cm}^{2}$. Deposition parameters were chosen to result in a low particle density, approximately 1 particle $/ 10^{4} \mu \mathrm{m}^{2}$ for particles of size between 50-1000 $\mathrm{nm}$ while that of smaller cluster $(<$ $50 \mathrm{~nm}$ ) was below the critical value to produce a continuous film (about 10\% of the surface is covered by these particles). This particular distribution of the particles has made possible the investigation of isolated single particles excluding any proximity interaction effect. Ultrathin 2$3 \mathrm{~nm} \mathrm{MgO}$ films grown on a W(110) have been used as substrate for the PtRh particles. Figure 4(a) shows a SPEM image of a typical sample; a single micron-sized $\mathrm{Pt}-\mathrm{Rh}$ particle is well visible due to the strong enhancement of the contrast induced by its morphology. The region surrounding the island appears uniform since it is partially covered by small cluster not resolvable with the SPEM spatial resolution.

Typical SPEM and SEM images of the micron-scale particles found on the surface are shown in Fig. 4(b). The identical shape-size-morphology information was provided by both $\mathrm{Rh} 3 d_{5 / 2}$ and $\mathrm{Pt} 4 f_{7 / 2}$ SPEM maps suggesting a stoichiometrical homogeneity of the probed regions. The contrast in the map is generated, in fact, by the main morphology of the surface and must not be attributed to changes in the chemical composition of the particle itself. Thanks to the higher lateral resolution, the SEM picture reveals not only the donut-like shape of the particles but also its polycrystalline structure. Despite the week contrast in the SEM detection induced by the structural changes appearing on the surface of the particle, in fact, the image clearly shows the presence of crystals with dimensions ranging from few tens to few hundred $\mathrm{nm}$. A more detailed characterization of the structural properties of these nano-crystals was performed with other investigation techniques and can be found in Ref. [15].

The $\mathrm{Rh} 3 d_{5 / 2}$ spectra acquired on points labeled as A and B of the particle in Fig. 4(b), taken after an oxidation cycle are shown in Fig. 4(c). The best deconvolution of these spectra requires several components corresponding to the following chemical states: metallic $\mathrm{Rh}$ at $307.2 \mathrm{eV}$ $\mathrm{BE}$ and $\mathrm{RhO}_{<1.5}, \mathrm{Rh}_{2} \mathrm{O}_{3}$ and $\mathrm{RhO}_{2}$ at $307.7 \mathrm{eV}, 308.2 \mathrm{eV}$ and $309.0 \mathrm{eV} \mathrm{BE}$, respectively. These spectra taken on the 'large' particles upon oxidation show sensible site dependence; in the centre of the particle (position A) there is still a weak metallic component and the oxidation did not proceed till the stable $\mathrm{RhO}_{2}$ phase as it happened for position B where almost $40 \%$ of the surface layers have been converted to rhodium dioxide. The present results show that changes may occur on very local microscopic scales resulting in compositional heterogeneity. The observed compositional complexity of the PtRh particles supports the most recent views that in catalytic reactions only fractions of the particle surface can behave as an active catalyst, i.e. looking for simple size-activity trends on assumption of a known equilibrium shape, structure and composition is far from the reality.

\section{CONCLUSIONS}

Recent achievements on imaging and probing the submicro objects have been presented in this report. For the first time, SPEM is used to explore the chemical state and chemical composition of individual $\mathrm{ZnO}$ nanostructures. The compositional homogeneity of the structures and high purity of the nanostructures were confirmed by SPEM measurements. The novel morphology and compositional homogeneity of the synthesized prickly $\mathrm{ZnO}$ nanoarchitectures make them a good candidate for use in various applications such as solar cells, superhydrophobic coatings, 
energy conversion/harvesting, chemical sensing, etc. The capability of SPEM on imaging an individual MWCNT, down to $50 \mathrm{~nm}$ in diameter is shown. The MWCNTs, partially covered with a metal, successfully are fabricated and presence of a sharp interface between CNT and metal is confirmed by SPEM. The morphology, composition and oxidation/reduction of isolated supported PtRh microand nano- particles produced by pulsed-laser deposition (PLD) have been investigated. The results show that changes occur on very local microscopic scales resulting in compositional heterogeneity. It believes that in catalytic reactions only fractions of the particle surface can behave as an active catalyst.

\section{Acknowledgments}

M. K. Abyaneh would like to thank S. K. Kulkarni for valuable and helpful discussions.
[1] S. Gunther, B. Kaulich, L. Gregoratti, and M. Kiskinova, Prog. Surf. Sci. 70, 187 (2002)

[2] A. Locatelli, L. Aballe, T.O. Mentes, M. Kiskinova and E. Bauer, Surf. Interface. Anal. 38, 1554 (2006).

[3] L. Gregoratti, A. Barinov, E. Benfatto, G. Cautero, C. Fava, P. Lacovig, D. Lonza, M. Kiskinova, R. Tommasini, and S. Mahl, Rev. Sci. Instrum. 75, 64 (2004).

[4] M. K. Abyaneh, S. Jafarkhani, L. Gregoratti, and S. K. Kulkarni, J. Nanoparticle Res. 13, 1311 (2010).

[5] S. A. Leontiev, S. V. Koshcheev, V. G. Devyatov, A. E. Cherkashin, and P. Mikheeva, J. Struc. Chem. 38, 725 (1997).

[6] J. Zhang, X. Liu, R. Blume, A. Zhang, R. Schlogl, and D.-S. Su, Science 322, 73 (2008).

[7] A. Felten, C. Bittencourt, and J. J. Pireaux, Nanotechnology 17, 1954 (2006).

[8] D. Tassis, N. Tagmatarchic, A. Bianco, and M. Prato,
Chem. Rev. 106, 1105 (2006).

[9] A. Barinov, L. Gregoratti, P. Dudin, S. La Rosa, and M. Kiskinova, Adv. Matter. 21, 1916 (2009).

[10] A. Barinov, H. Ustunel, S. Fabris, L. Gregoratti, L. Aballe, P. Dudin, S. Baroni, and M. Kiskinova, Phys. Rev. Lett. 99, 046803 (2007).

[11] Nanolab, Inc., see the arrays at http://www.nanolab.com.

[12] A. Baraldi, et al., J. Am. Chem. Soc. 127, 5671 (2005).

[13] P. Dudin, A. Barinov, L. Gregoratti, D. Scaini, Y. B. He, H. Over, and M. Kiskinova, J. Phys. Chem. C 112, 9040 (2008).

[14] J. Libuda and H. J. Freund, Surf. Sci. Rep. 57, 157 (2005).

[15] M. Dalmiglio, M. Amati, L. Gregoratti, T. O. Mentes, M. A. Nino, L. Felisari, and M. Kiskinova, J. Phys. Chem. C 114, 16885 (2010). 